\title{
The importance of the lepidic component as a prognostic factor in stage I pulmonary adenocarcinoma
}

\author{
Youngkyu Moon ${ }^{1}$, Sook Whan Sung ${ }^{1}$, Kyo Young Lee ${ }^{2}$, Young Kyoon Kim³ and Jae Kil Park ${ }^{\text {* }}$
}

\begin{abstract}
Background: Stage I pulmonary adenocarcinoma (PA) can offer an unfavorable prognosis. The aim of this study was to classify the prognosis of stage I PA on the basis of the lepidic component and to confirm whether the lepidic component can be used as a criterion for predicting the prognosis of stage I PA.

Methods: We conducted a retrospective study of patients who underwent curative surgery for stage I and IIA PA. Stage I disease was divided into three groups on the basis of the lepidic component: group 1, $\leq 10 \%$; group 2, >10 to $50 \%$; and group 3,>50 \%. We compared recurrence-free survival (RFS) rates among groups 1, 2, and 3, and stage IIA disease. We also evaluated risk factors for disease recurrence with multivariate analysis.

Results: A total of 224 patients were included in our study; most patients $(n=201)$ had stage I disease. Three-year RFS rates in group $1(n=73)$, group $2(n=75)$, and group $3(n=53)$ were $74.1,90.4$, and $90.0 \%$, respectively. There was a significant difference in RFS between group 1 and group $2(p=0.009)$. The 3 -year RFS rate in stage IIA disease was $61.4 \%$. There were no significant differences in RFS between group 1 and stage IIA disease $(p=0.163)$. In multivariate analysis, group 1 had the highest risk of recurrence (HR 5.806, $p=0.006$ ) in stage I PA.

Conclusions: Stage I PA with a lepidic component $\leq 10 \%$ was associated with an unfavorable prognosis that was similar to the prognosis of stage IIA disease. The prognosis for stage I PA should not be based on general criteria, but instead, the lepidic component should be evaluated and considered when determining disease prognosis.
\end{abstract}

Keywords: Lung cancer, Adenocarcinoma, Lepidic, Prognosis, Stage I, Recurrence, Adenocarcinoma classification

\section{Background}

Pulmonary adenocarcinoma (PA) is the most common histologic type of primary lung cancer [1]. In 2011, the International Association for the Study of Lung Cancer (IASLC), the American Thoracic Society (ATS), and the European Respiratory Society (ERS) proposed new subtypes of adenocarcinoma. According to the new classification system, the components of adenocarcinoma were classified into five major histopathologic categories on the basis of the growth pattern or shape of the tumor: acinar, papillary, micropapillary, solid, and lepidic [2]. Among the subtypes of PA, lepidic-predominant adenocarcinoma has the most favorable prognosis, and

\footnotetext{
* Correspondence: jaekpark@catholic.ac.kr

1Department of Thoracic \& Cardiovascular Surgery, Seoul St. Mary's Hospital, College of Medicine, The Catholic University of Korea, 222 Banpo-daero, Seocho-gu, Seoul 06591, Republic of Korea

Full list of author information is available at the end of the article
}

micropapillary-predominant and solid-predominant adenocarcinomas have unfavorable prognoses [2, 3]. However, in more than $90 \%$ of PA cases, two or more components are present and the prognosis of PA is complicated and varies on the basis of the ratio of each histopathologic component. After the adoption of the new classification system, many studies reported correlations between subtypes and histopathologic components of PA and disease prognosis [3-8].

Until now, the most important prognostic factor in non-small cell lung cancer (NSCLC) has been its anatomical stage [9]. However, recurrence can occur even with the most favorable prognosis in stage I, which indicates that other factors may affect prognosis and that the prognosis of stage I disease varies with histopathologic subtype [10]. Additionally, one study reported that the prognosis could vary with the respective occupancy 
rates of the five histopathologic components [5]. Therefore, stage I PA may be able to be divided into favorable prognosis and unfavorable prognosis, which would help to predict the disease course for patients with stage I PA. The lepidic component is the most common component among the five histopathologic components in stage I PA, so it is reasonable to classify stage I PA on the basis of the occupancy rate of the lepidic component.

The aim of this study was to confirm the usefulness of classifying the prognosis of stage I PA on the basis of the occupancy rate of the lepidic component. Further, we aimed to evaluate whether the lepidic component could be used to predict the prognosis of PA.

\section{Methods}

\section{Patients}

We conducted a retrospective chart review of 265 patients who were diagnosed with stage I and stage IIA PA and underwent curative resection at Seoul St. Mary's Hospital at the Catholic University of Korea between August 2010 and December 2013. Among 242 patients with stage I PA, 41 patients were excluded from the study: 25 patients who were diagnosed with adenocarcinoma in situ (AIS) or minimally invasive adenocarcinoma (MIA), 15 patients who were diagnosed with mucinous adenocarcinoma, and 1 patient who was diagnosed with adenocarcinoma with neuroendocrine features. Mucinous adenocarcinoma was excluded from our study because it exhibits clinical, pathologic, and genetic differences from non-mucinous adenocarcinoma and it is classified in a new category of "variants of invasive adenocarcinoma" in the IASLC/ATS/ERS classification system [2]. A total of 224 patients were included in the final study analysis: 201 patients with stage I PA and 23 patients with stage IIA PA. Cancer staging was determined according to the TNM classification system defined by the American Joint Committee on Cancer [9]. This study was approved by the institutional review board of Seoul St. Mary's Hospital at the Catholic University of Korea.

\section{Histologic evaluation}

All clinical specimens were examined by pathology specialists, and their observations were recorded in pathology records. For the description of histopathologic components, the occupancy ratio of each component (lepidic, acinar, papillary, micropapillary, and solid) in the total tumor area was measured and recorded in $5 \%$ increments, according to the 2011 IASLC/ATS/ERS classification system [2]. The 201 patients with stage I disease were divided into three groups on the basis of the occupancy ratio of the lepidic component in the total tumor: group $1, \leq 10 \%$; group $2,>10$ to $50 \%$; and group $3,>50 \%$.

\section{Statistical analysis}

We divided stage I disease into three groups according to the percentage of the lepidic component, and we compared the clinicopathologic characteristics among the groups. We also compared characteristics between stage I and stage IIA disease and group 1 and stage IIA disease. For these comparisons, we used Student's $t$ test for continuous variables and the $\chi^{2}$ test for categorical variables. We used the analysis of variance test and the Kruskal-Wallis $\mathrm{H}$ test to compare the means of the groups. We analyzed data from the time of surgery to the last date of follow-up, and we determined recurrence-free survival (RFS) using the Kaplan-Meier method by calculating the cases of recurrence and death. We compared survival rates among the groups using log rank statistics. We used the Cox proportional hazards model for the multivariate analysis to determine risk factors for recurrence in patients with stage I disease. A value of $p<0.05$ was considered statistically significant.

\section{Results}

The mean age of all 224 patients (stages I and IIA) was 63.7 years (range, 38-85 years), and more than half (58.9\%) of the patients were female. Stage I disease was divided into groups 1,2, and 3 according to the lepidic component; the clinical characteristics of each group are presented in Table 1 . Group 1 contained more male patients $(60.3 \%)$ than female patients, but groups 2 and 3 contained more female patients than male patients $(p<0.001)$. Mean smoking amount of group 1 was significantly higher than that of group 2 but was not significantly different from that of group 3 . There were no differences in age, sex, or smoking history between patients with stage I and stage IIA disease. The maximum standardized uptake value (SUVmax) of fluorodeoxyglucose in positron emission tomography (PET) in group 1 was significantly higher than that of group 3 but was not different than that of group 2. The SUVmax in stage IIA disease was significantly higher than that in stage I disease $(p<0.001)$. The SUVmax in stage IIA disease was also significantly higher than that in group 1 (8.8 vs. 4.5; $p<0.001$ ). There was no difference in the rate of pulmonary resection among groups 1,2 , and 3 or between stage I and stage IIA disease. Mediastinal lymph node dissection or sampling was conducted for every patient; there were no differences in the average number of removed lymph nodes among groups 1,2 , and 3 , but there was a small difference between stage I and IIA disease $(13.5 \pm 9.5$ vs. $17.7 \pm 8.5$, respectively; $p=0.043$ ).

The pathologic characteristics of patients with stage I PA, according to the lepidic component, are presented in Table 2 . There was no difference in average tumor size $(p=0.076)$. The incidence of pleural invasion and vascular invasion were higher in groups 1 
Table 1 Clinical characteristics of patients with pulmonary adenocarcinoma, according to disease stage and lepidic component

\begin{tabular}{|c|c|c|c|c|c|c|c|}
\hline & Stage I & & & & & Stage $\| \mathrm{A}$ & $p$ value \\
\hline & Total & Group 1 & Group 2 & Group 3 & $\overline{p \text { value }}{ }^{a}$ & $(n=23)$ & \\
\hline & $(n=217)$ & $(n=73)$ & $(n=75)$ & $(n=53)$ & & & \\
\hline Age, years $( \pm S D)$ & $64.0( \pm 9.3)$ & $64.5( \pm 10.3)$ & $65.2( \pm 9.4)$ & $61.8( \pm 7.4)$ & 0.121 & $61.0( \pm 7.5)$ & 0.131 \\
\hline Sex & & & & & $<0.001$ & & 0.518 \\
\hline Female, $n(\%)$ & $117(58.2 \%)$ & 29 (39.7 \%) & $59(78.7 \%)$ & $29(54.7 \%)$ & & $15(65.2)$ & \\
\hline Male, $n(\%)$ & $84(41.8 \%)$ & $44(60.3 \%)$ & $16(21.3 \%)$ & $24(45.3 \%)$ & & $8(34.8)$ & \\
\hline Smoking, pack years $( \pm S D)$ & $8.6( \pm 15.6)$ & $14.2( \pm 19.1)$ & $3.8( \pm 10.9)$ & $7.3( \pm 13.3)$ & $<0.001$ & $5.7( \pm 14.7)$ & 0.424 \\
\hline Laterality & & & & & 0.469 & & 0.038 \\
\hline Right, $n(\%)$ & $137(68.2 \%)$ & $47(64.4 \%)$ & $55(73.3 \%)$ & $35(66.0 \%)$ & & $10(43.5 \%)$ & \\
\hline Left, $n(\%)$ & $64(31.8 \%)$ & 26 (35.6 \%) & $20(26.7 \%)$ & $18(34.0 \%)$ & & $13(56.5 \%)$ & \\
\hline SUVmax $( \pm S D)$ & $3.6( \pm 3.2)$ & $4.5( \pm 3.8)$ & $3.7( \pm 3.0)$ & $2.0( \pm 1.8)$ & $<0.001$ & $8.8( \pm 3.7)$ & $<0.001$ \\
\hline Extent of operation & & & & & 0.416 & & 0.273 \\
\hline Wedge resection, $n(\%)$ & $17(8.5 \%)$ & $8(11.0 \%)$ & $5(6.7 \%)$ & $4(7.5 \%)$ & & 0 & \\
\hline Segmentectomy, $n$ (\%) & $10(5.0 \%)$ & $2(2.7 \%)$ & $6(8.0 \%)$ & $2(3.8 \%)$ & & 0 & \\
\hline Lobectomy, n (\%) & $170(84.6 \%)$ & 62 (84.9\%) & $61(81.3 \%)$ & 47 (88.7 \%) & & 22 (95.7\%) & \\
\hline Bilobectomy, n (\%) & 4 (2.0\%) & 1 (1.4\%) & $3(4.0 \%)$ & 0 & & 1 (4.3\%) & \\
\hline Surgical approach & & & & & 0.475 & & 0.692 \\
\hline VATS, $n(\%)$ & $178(88.6 \%)$ & 62 (84.9\%) & 68 (90.7 \%) & $48(90.6 \%)$ & & 21 (91.3\%) & \\
\hline Thoracotomy, n (\%) & $21(11.4 \%)$ & $11(15.1 \%)$ & 7 (9.3\%) & $5(9.4 \%)$ & & 2 (8.7\%) & \\
\hline Sampled lymph node, $n( \pm S D)$ & $13.5( \pm 9.5)$ & $14.0( \pm 10.7)$ & $14.0( \pm 9.2)$ & $12.3( \pm 7.9)$ & 0.537 & $17.7( \pm 8.5)$ & 0.043 \\
\hline
\end{tabular}

Group 1 = lepidic component $\leq 10 \%$; group 2 = lepidic component $>10$ to $50 \%$; group $3=$ lepidic component $>50 \%$

SD standard deviation, SUVmax maximum standardized uptake value of fluorodeoxyglucose, VATS video-assisted thoracoscopic surgery

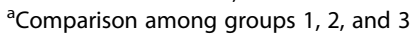

${ }^{\mathrm{b} C o m p a r i s o n}$ between stage I and stage IIA

and 2 than in group 3. The occupancy ratios of micropapillary and acinar components were similar in groups 1 and 2, and the occupancy ratios of solid and papillary components were significantly higher in group 1 than in groups 2 and 3.
The pathologic characteristics of patients in group 1 and patients with stage IIA disease are presented in Table 3. Patients with stage IIA disease had a larger tumor size than patients in group $1(3.0$ vs. $2.2 \mathrm{~cm}$; $p=0.005$ ), as well as higher rates of lymphatic invasion

Table 2 Pathologic characteristics of patients with stage I pulmonary adenocarcinoma according to lepidic component

\begin{tabular}{|c|c|c|c|c|}
\hline & Group 1 & Group 2 & Group 3 & $p$ value \\
\hline Tumor size, $\mathrm{cm}( \pm \mathrm{SD})$ & $2.2( \pm 0.9) \mathrm{cm}$ & $2.3( \pm 0.9) \mathrm{cm}$ & $2.0( \pm 0.7) \mathrm{cm}$ & 0.076 \\
\hline Pleural invasion, $n(\%)$ & $23(31.9 \%)$ & $18(24.3 \%)$ & $4(7.5 \%)$ & 0.005 \\
\hline Lymphatic invasion, $n$ (\%) & $21(29.2 \%)$ & $28(37.8 \%)$ & $9(17.0 \%)$ & 0.039 \\
\hline Vascular invasion, n (\%) & $9(12.5 \%)$ & $9(12.5 \%)$ & $1(1.9 \%)$ & 0.088 \\
\hline Differentiation & & & & $<0.001$ \\
\hline Mild, $n(\%)$ & 15 (20.5\%) & $41(54.7 \%)$ & $48(90.6 \%)$ & \\
\hline Moderate, $n(\%)$ & 49 (67.1\%) & $31(41.3 \%)$ & 5 (9.4\%) & \\
\hline Poor, n (\%) & $9(12.3 \%)$ & $3(4.0 \%)$ & $0(0 \%)$ & \\
\hline EGFR mutation & $75.4 \%$ & $90.3 \%$ & $79.4 \%$ & 0.087 \\
\hline Micropapillary component, \% ( \pm SD) & $3.4( \pm 9.4) \%$ & $3.0( \pm 8.5) \%$ & $0.1( \pm 0.7) \%$ & 0.006 \\
\hline Solid component, \% ( \pm SD) & $15.2( \pm 27.9) \%$ & $1.1( \pm 3.7) \%$ & $0.3( \pm 1.6) \%$ & $<0.001$ \\
\hline Acinar component, \% ( \pm SD) & $49.0( \pm 37.7) \%$ & $52.2( \pm 21.6) \%$ & $21.0( \pm 11.2) \%$ & $<0.001$ \\
\hline Papillary component, \% ( \pm SD) & $23.8( \pm 22.5) \%$ & $8.7( \pm 18.2) \%$ & $2.9( \pm 6.8) \%$ & $<0.001$ \\
\hline
\end{tabular}

Group 1 = lepidic component $\leq 10 \%$; group 2 = lepidic component $>10$ to $50 \%$; group 3 = lepidic component $>50 \%$ EGFR epidermal growth factor receptor, SD standard deviation 
Table 3 Comparison of pathologic characteristics between patients in group 1 and patients with stage IIA pulmonary adenocarcinoma

\begin{tabular}{llll}
\hline & Group 1 & Stage IIA & $p$ value \\
\hline Tumor size, cm $( \pm$ SD) & $2.2( \pm 0.9) \mathrm{cm}$ & $3.0( \pm 1.3) \mathrm{cm}$ & 0.005 \\
Pleural invasion, $n(\%)$ & $23(31.9 \%)$ & $9(40.9 \%)$ & 0.298 \\
Lymphatic invasion, $n(\%)$ & $21(29.2 \%)$ & $20(87.0 \%)$ & $<0.001$ \\
Vascular invasion, $n(\%)$ & $9(12.5 \%)$ & $11(47.8 \%)$ & 0.001 \\
Differentiation & & & 0.534 \\
$\quad$ Mild, $n(\%)$ & $15(20.5 \%)$ & $4(17.4 \%)$ & \\
$\quad$ Moderate, $n(\%)$ & $49(67.1 \%)$ & $14(60.9 \%)$ & \\
$\quad$ Poor, $n(\%)$ & $9(12.3 \%)$ & $5(21.7 \%)$ & \\
EGFR mutation & $75.4 \%$ & $76.2 \%$ & 0.597 \\
Micropapillary component, & $3.4( \pm 9.4) \%$ & $5.5( \pm 8.9) \%$ & 0.378 \\
\% ( \pm SD) & & & \\
Solid component, \% $( \pm$ SD) & $15.1( \pm 27.9) \%$ & $15.7( \pm 29.5) \%$ & 0.936 \\
Acinar component, \% $( \pm$ SD) & $49.0( \pm 37.7) \%$ & $48.3( \pm 28.5) \%$ & 0.927 \\
Papillary component, \% $( \pm$ SD) & $23.84( \pm 33.5) \%$ & $17.1( \pm 22.7) \%$ & 0.296 \\
Lepidic component, \% $( \pm$ SD) & $4.4( \pm 4.6) \%$ & $9.9( \pm 15.0) \%$ & 0.095 \\
\hline
\end{tabular}

Group 1 = stage I disease with lepidic component $\leq 10 \%$

EGFR epidermal growth factor receptor, $S D$ standard deviation

and vascular invasion $(p<0.001$ and $p=0.001$, respectively). However, there were no significant differences in the occupancy ratios of histopathologic components of the tumor between these two groups. Specifically, the lepidic component was not different between group 1 and stage IIA disease (4.4 vs. $9.9 \%$, respectively; $p=0.095$ ).

The median follow-up time for all patients was 720 days (range, 12-1564 days). During the follow-up period, disease recurrence occurred in 22 (10.9 \%) patients with stage I PA and 7 (30\%) patients with stage IIA disease. The 3-year RFS rate in stage I disease was significantly higher than in stage IIA disease (86.3 vs. $61.4 \%$, respectively; $p=0.001$ ) (Fig. 1). The 3-year RFS rates in groups 1, 2, and 3 were 74.1, 90.4, and $90.0 \%$, respectively (Fig. 2). The RFS in group 2 was significantly higher than that in group $1(p=0.009)$, but it was not different than that in group 3. There was no difference in 3-year RFS between group 1 and stage IIA disease ( 74.1 vs. $61.4 \%$, respectively; $p=0.163$ ).

The risk factors for recurrence according to multivariate analysis are presented in Table 4. We analyzed the respective effect of each of the five histopathologic components (\%) on disease recurrence using covariate factors of age, sex, smoking history, SUVmax, tumor size, and rates of pleural invasion, lymphatic invasion, and vascular invasion. Only the lepidic component was significantly related to recurrence (hazard ratio $(\mathrm{HR})=0.955,95 \% \mathrm{CI}=0.924-0.987$; $p=0.007$ ). A lepidic component of $\leq 10 \%$ (group 1 ) was associated with the highest risk of recurrence $(\mathrm{HR}=5.806$, $95 \% \mathrm{CI}=1.666-20.238 ; p=0.006$ ).

\section{Discussion}

Most cases of PA display a mixture of five histopathologic components. In the early stages of the disease, the lepidic component is the most common component. In our study, we divided stage I disease into three relatively even groups on the basis of lepidic component $(n=73$, 75, and 53). The 3-year RFS rates of the three groups were $74.1,90.4$, and $90.0 \%$, and we demonstrated that a higher proportion of lepidic component in the tumor was associated with better survival. RFS was lowest with a lepidic component $\leq 10 \%$, and the prognosis for patients with stage I disease with a lepidic component $\leq 10 \%$ was not better than the prognosis for patients with more advanced disease.

According to the 2011 IASLC/ATS/ERS classification criteria, acinar, papillary, micropapillary, and solid components are classified as invasive components and differentiated from lepidic components [2]. Therefore, a higher proportion of invasive components is associated with easier tumor invasion and an increased likelihood of tumor progression. Consequently, the relative amounts of components other than the lepidic component could be important factors in determining prognosis. In the case of stage I disease, the occupancy ratio of the lepidic component is so high that the occupancy ratios of the other components are relatively low. In our study, groups 1 and 2 showed different survival rates, and an evaluation of the different components present in the tumors of these groups indicated that only solid and papillary components were higher in group 1 and that there were no differences in the ratios of acinar or micropapillary components. Many studies have reported that only micropapillary and lepidic components are independently related to disease recurrence, and few studies have reported that acinar, solid, or papillary components individually affect prognosis $[4,5,11,12]$. Since we observed no difference in the proportion of micropapillary component between groups 1 and 2, we conclude that the difference in survival was due to the difference in lepidic component and not a difference in solid or papillary components. Further, since the results of our multivariate analysis indicated that only the lepidic component was found to be a risk factor for recurrence, the low proportion of lepidic component could be emphasized as a significant risk factor for disease recurrence.

In NSCLC, stage IA and IB disease are differentiated by tumor size and the presence of visceral pleural invasion [9] and stage IA disease has a higher survival rate than stage IB disease [13-15]. However, in addition to tumor size and visceral pleural invasion that differentiate stages IA and IB, many other factors are being investigated that might influence disease prognosis, including histopathologic components. In our study, we included tumor size and visceral pleural invasion as covariate 


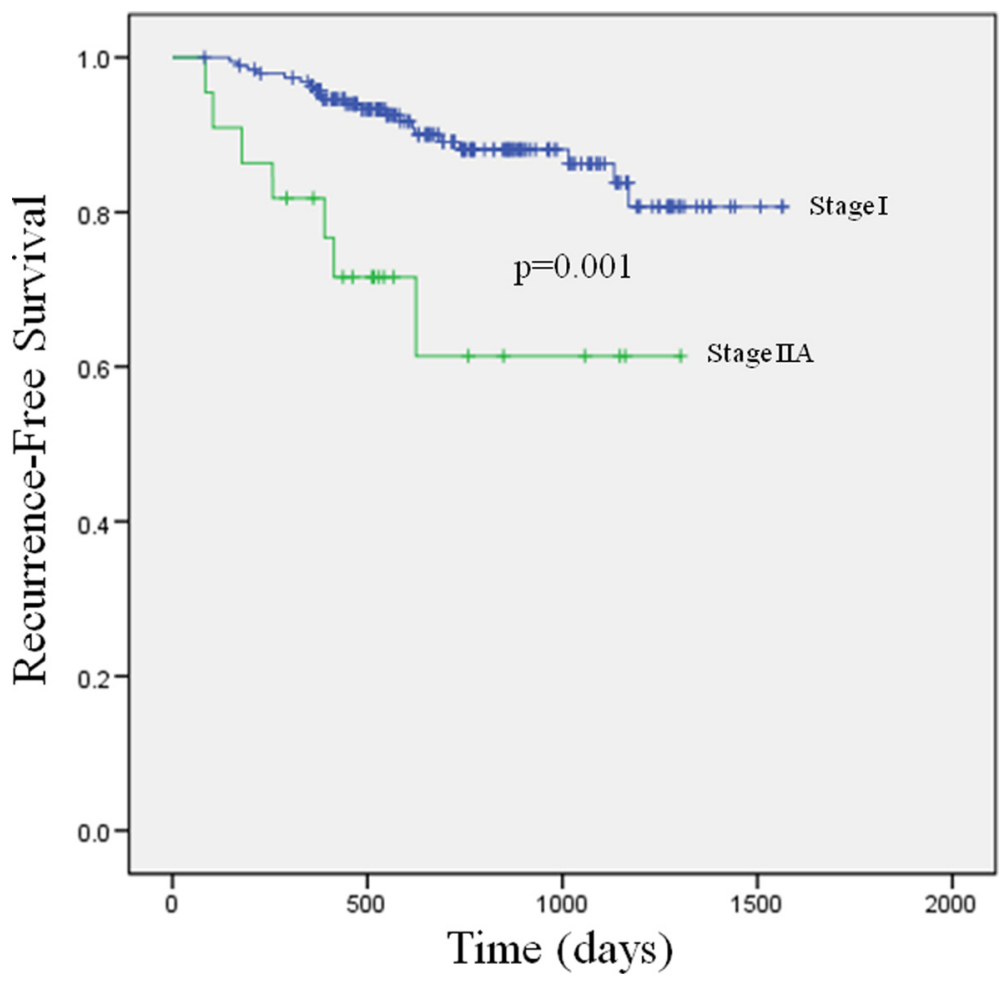

Fig. 1 Three-year recurrence-free survival of patients with stage I and stage IIA pulmonary adenocarcinoma

factors in the multivariate analysis and the percentage of lepidic component was found to influence prognosis. In group 1, specifically, a low percentage of lepidic component was determined to be a risk factor for recurrence with an HR of 5.8. Therefore, this criterion could be used to determine prognosis as well as differentiate stage IA from stage IB disease.

When we examined the clinicopathologic characteristics of group 1, we observed that this group contained more males and more people with a smoking history than groups 2 and 3, as well as stage IIA disease. It shows that the characteristics of patients in group 1 were different than those of patients with other types of adenocarcinoma. We observed no significant differences between groups 1 and 2 when we evaluated other known prognostic factors such as SUVmax, tumor size, percentage of micropapillary component, and rates of pleural invasion, lymphatic invasion, and vascular invasion [3, 16-20]. However, there was a difference in RFS between groups 1 and 2, which indicated that the difference in prognosis was due to the difference in the lepidic component between the groups.

The lepidic component represents only a small proportion of tumors in stage II disease, so it is only possible to differentiate stage I disease according to the lepidic component [5]. The results of our comparison of clinicopathologic characteristics between group 1 and stage IIA disease showed that there was significantly more lymphatic invasion and vascular invasion and tumor size was larger in stage IIA disease. Tumor size and lymphovascular invasion are important prognostic factors for PA [16-19]. SUVmax, which is a known prognostic factor in early lung cancer, was also significantly higher in stage IIA disease [3, 20]. Moreover, $21(91.3 \%)$ patients with stage IIA disease had lymph node metastasis. On the basis of these results alone, the prognosis of stage IIA could be assumed to be worse than that of group 1. However, we observed no difference in the distributions of histopathologic components or 3-year RFS between group 1 and stage IIA disease. Therefore, we inferred that the prognosis of stage I disease could be similar to that of stage IIA disease if the histopathologic components showed similar patterns, even if the pathologic features are worse in stage IIA disease.

In this study, we evaluated RFS instead of overall survival because, in the case of stage I disease, more patients die from other causes than from the relevant cancer during the follow-up period [3]. Also, RFS is a more accurate measurement of survival analysis, since it reflects the biological behavior of the cancer rather than death due to unrelated factors. Further, since we only included relatively recent data in our analysis, we were only able to use 3-year survival data to evaluate disease prognosis. This restriction was necessary because the 


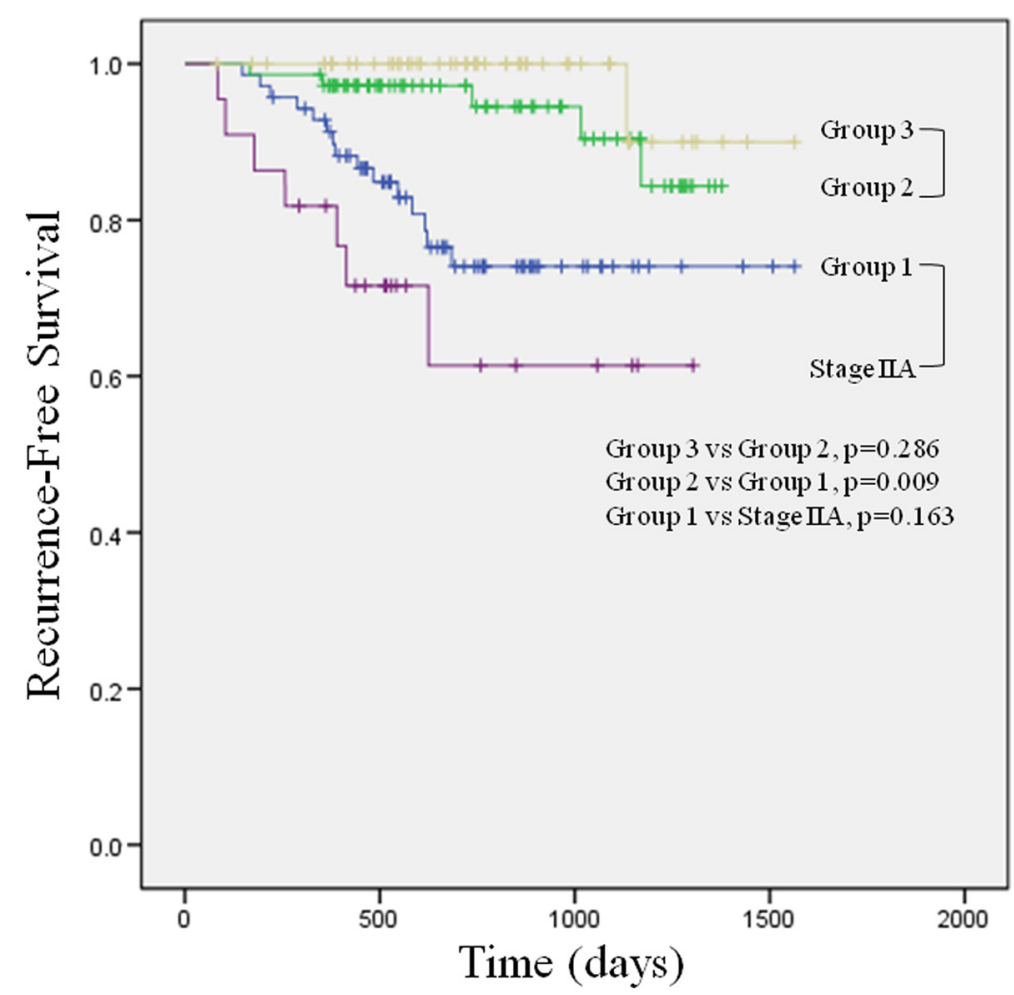

Fig. 2 Three-year recurrence-free survival among three groups of patients with stage I pulmonary adenocarcinoma and patients with stage IIA pulmonary adenocarcinoma. Group 1 = stage I disease with lepidic component $\leq 10 \%$; group 2 = stage I disease with lepidic component $>10 \%$ to $50 \%$; group 3 = stage I disease with lepidic component $>50 \%$

Table 4 Multivariate analysis of risk factors for recurrence of pulmonary adenocarcinoma

\begin{tabular}{lllc}
\hline & HR & $95 \%$ Cl & $p$ value \\
\hline Age & 0.960 & $0.911-1.011$ & 0.120 \\
Sex & 0.678 & $0.161-2.853$ & 0.597 \\
Smoking history & 1.006 & $0.974-1.039$ & 0.714 \\
SUVmax & 1.011 & $0.860-1.189$ & 0.893 \\
Tumor size & 1.456 & $0.715-2.965$ & 0.300 \\
Pleural invasion & 0.469 & $0.117-1.874$ & 0.284 \\
Lymphatic invasion & 2.487 & $0.677-9.134$ & 0.170 \\
Vascular invasion & 1.496 & $0.321-6.960$ & 0.608 \\
Lepidic component (\%) & 0.955 & $0.924-0.987$ & 0.007 \\
Micropapillary component (\%) & 1.040 & $0.991-1.092$ & 0.114 \\
Acinar component (\%) & 1.012 & $0.993-1.032$ & 0.209 \\
Papillary component (\%) & 1.016 & $0.994-1.038$ & 0.155 \\
Solid component (\%) & 0.982 & $0.927-1.041$ & 0.546 \\
Lepidic component $\leq 10 \%$ & 5.806 & $1.666-20.238$ & 0.006 \\
\hline
\end{tabular}

$\mathrm{Cl}$ confidence interval, $H R$ hazard ratio, SUVmax maximum standardized uptake value of fluorodeoxyglucose institution at which the authors practice established video-assisted thoracoscopic surgery (VATS) as the general method of operation beginning in August 2010; since that time, this type of operation has been performed on the majority of patients at the institution and full mediastinal lymph node dissection has been performed. In addition, we determined that 3-year RFS was sufficient to examine prognosis since, in PA, most cases of postoperative recurrence occur within 2 years and cancer that recurs after 2 years has a high likelihood of being metachronous cancer [21].

This study has several limitations that should be considered. First, we used a retrospective study design. Second, we obtained the data from a single institution and the number of cases was relatively small. However, most studies that have evaluated the histopathologic classification of stage I PA included approximately 200 cases. Compared to these studies, the 217 patients with stage I disease included in our study do not comprise a small sample size [10, 22-27]. Of course, more accurate results could be obtained if the analysis and comparison were made with a larger patient sample. Third, we used recent data (2010 to 2013), so only short-term follow-up information was available and only 3-year survival evaluation was possible. However, as mentioned, most cases 
of recurrence of lung cancer occur within 2 years, and we judged that 3-year RFS was suitable for the determination of prognosis [21]. More accurate results could be obtained if more data were available from progressive observations over a longer period of time.

\section{Conclusions}

In conclusion, recurrence often occurs in stage I PA, even after curative surgery. Specifically, recurrence is likely in cases in which the lepidic component of the tumor is less than or equal to $10 \%$. Further, in stage I disease with a low lepidic component, recurrence rates are not significantly different than those of stage IIA disease and generally applied stage I prognoses should not be expected. Adjuvant treatment, which is often performed in stage II disease, can be considered for stage I disease with a low lepidic component. Future studies that collect data from larger sample sizes will confirm the relationships between the histopathologic components of PA and disease prognosis.

\section{Abbreviations}

ATS: American Thoracic Society; ERS: European Respiratory Society: HR: hazard ratio; IASLC: International Association for the Study of Lung Cancer; NSCLC: non-small cell lung cancer; PA: pulmonary adenocarcinoma; PET: positron emission tomography; RFS: recurrence-free survival; SUVmax: maximum standardized uptake value; VATS: video-assisted thoracoscopic surgery.

\section{Competing interests}

The authors declare that they have no competing interests.

\section{Authors' contributions}

YM participated in the design of the study, performed the statistical analysis, and drafted the manuscript. SWS, KYL, and YKK participated in the design of the study. JKP conceived of the study, participated in its design and coordination, and helped to draft the manuscript. All authors read and approved the final manuscript.

\section{Acknowledgements}

This research was not supported by outside funding.

This article has been edited by native English-speaking experts of BioMed Proofreading, LLC.

\section{Author details \\ 'Department of Thoracic \& Cardiovascular Surgery, Seoul St. Mary's Hospital, College of Medicine, The Catholic University of Korea, 222 Banpo-daero, Seocho-gu, Seoul 06591, Republic of Korea. ${ }^{2}$ Department of Hospital Pathology, Seoul St. Mary's Hospital, College of Medicine, The Catholic University of Korea, Seoul, Republic of Korea. ${ }^{3}$ Department of Internal Medicine, Seoul St. Mary's Hospital, College of Medicine, The Catholic University of Korea, Seoul, Republic of Korea.}

Received: 25 August 2015 Accepted: 11 February 2016

Published online: 16 February 2016

\section{References}

1. Parkin DM, Ferlay J, Curado MP, Bray F, Edwards B, Shin HR, et al. Fifty years of cancer incidence: CI5 HIX. Int J Cancer. 2010;127(12):2918-27. doi:10.1002/ijc.25517.

2. Travis WD, Brambilla E, Noguchi M, Nicholson AG, Geisinger KR, Yatabe Y, et al. International Association for the Study of Lung Cancer/American Thoracic Society/European Respiratory Society international multidisciplinary classification of lung adenocarcinoma. J Thorac Oncol. 2011:6(2):244-85. doi:10.1097/JTO.0b013e318206a221.
3. Eguchi T, Kadota K, Park BJ, Travis WD, Jones DR, Adusumilli PS. The new IASLC-ATS-ERS lung adenocarcinoma classification: what the surgeon should know. Semin Thorac Cardiovasc Surg. 2014;26(3):210-22. doi:10.1053/j.semtcvs.2014.09.002.

4. Zhang Y, Wang R, Cai D, Li Y, Pan Y, Hu H, et al. A comprehensive investigation of molecular features and prognosis of lung adenocarcinoma with micropapillary component. J Thorac Oncol. 2014;9(12):1772-8. doi:10.1097/JTO.0000000000000341.

5. Moon Y, Kim KS, Sung SW, Lee KY, Kim YK, Kang JH, et al. Correlation of histological components with tumor invasion in pulmonary adenocarcinoma. World J Surg Oncol. 2014;12:388. doi:10.1186/1477-7819-12-388.

6. Kadota K, Villena-Vargas J, Yoshizawa A, Motoi N, Sima CS, Riely GJ, et al. Prognostic significance of adenocarcinoma in situ, minimally invasive adenocarcinoma, and nonmucinous lepidic predominant invasive adenocarcinoma of the lung in patients with stage I disease. Am J Surg Pathol. 2014;38(4):448-60. doi:10.1097/pas.0000000000000134.

7. Chao L, Yi-Sheng H, Yu C, Li-Xu Y, Xin-Lan L, Dong-Lan L, et al. Relevance of EGFR mutation with micropapillary pattern according to the novel IASLC/ATS/ERS lung adenocarcinoma classification and correlation with prognosis in Chinese patients. Lung Cancer. 2014;86(2):164-9. doi:10.1016/j.lungcan.2014.08.018.

8. Westaway DD, Toon CW, Farzin M, Sioson L, Watson N, Brady PW, et al. The International Association for the Study of Lung Cancer/American Thoracic Society/European Respiratory Society grading system has limited prognostic significance in advanced resected pulmonary adenocarcinoma. Pathology. 2013;45(6):553-8. doi:10.1097/PAT.0b013e32836532ae.

9. Edge SB, Byrd DR, Compton CC, Fritz AG, Greene FL, Trotti A. AJCC cancer staging manual. 7th ed. New York: Springer; 2010.

10. Zhang J, Wu J, Tan Q, Zhu L, Gao W. Why do pathological stage IA lung adenocarcinomas vary from prognosis?: a clinicopathologic study of 176 patients with pathological stage IA lung adenocarcinoma based on the IASLC/ATS/ERS classification. J Thorac Oncol. 2013;8(9):1196-202. doi:10.1097/JTO.0b013e31829f09a7.

11. Sumiyoshi S, Yoshizawa A, Sonobe M, Kobayashi M, Fujimoto M, Tsuruyama T, et al. Pulmonary adenocarcinomas with micropapillary component significantly correlate with recurrence, but can be well controlled with EGFR tyrosine kinase inhibitors in the early stages. Lung Cancer. 2013;81(1):53-9. doi:10.1016/j. lungcan.2013.04.003.

12. Zhang J, Liang Z, Gao J, Luo Y, Liu T. Pulmonary adenocarcinoma with a micropapillary pattern: a clinicopathological, immunophenotypic and molecular analysis. Histopathology. 2011;59(6):1204-14. doi:10.1111/j.1365-2559.2011.04050.x.

13. Suzuki M, Yoshida S, Tamura H, Wada H, Moriya Y, Hoshino H, et al. Applicability of the revised International Association for the Study of Lung Cancer staging system to operable non-small-cell lung cancers. Eur J Cardiothorac Surg. 2009;36(6):1031-6. doi:10.1016/j.ejcts.2009.06.025.

14. Kameyama K, Takahashi M, Ohata K, Igai H, Yamashina A, Matsuoka T, et al. Evaluation of the new TNM staging system proposed by the International Association for the Study of Lung Cancer at a single institution. J Thorac Cardiovasc Surg. 2009;137(5):1180-4. doi:10.1016/j.jtcvs.2008.09.030.

15. Fibla JJ, Cassivi SD, Decker PA, Allen MS, Darling GE, Landreneau RJ, et al. Validation of the lung cancer staging system revisions using a large prospective clinical trial database (ACOSOG Z0030). Eur J Cardiothorac Surg. 2013;43(5):911-4. doi:10.1093/ejcts/ezs520.

16. Nentwich MF, Bohn BA, Uzunoglu FG, Reeh M, Quaas A, Grob TJ, et al. Lymphatic invasion predicts survival in patients with early node-negative non-small cell lung cancer. J Thorac Cardiovasc Surg. 2013;146(4):781-7. doi:10.1016/j.jtcvs.2013.04.037.

17. Mimae $T$, Tsutani $Y$, Miyata $Y$, Yoshiya $T$, Ibuki $Y$, Kushitani $K$, et al. Role of lymphatic invasion in the prognosis of patients with clinical node-negative and pathologic node-positive lung adenocarcinoma. J Thorac Cardiovasc Surg. 2014;147(6):1820-6. doi:10.1016/j.jtcvs.2013.11.050.

18. Funai K, Sugimura H, Morita T, Shundo Y, Shimizu K, Shiiya N. Lymphatic vessel invasion is a significant prognostic indicator in stage IA lung adenocarcinoma. Ann Surg Oncol. 2011;18(10):2968-72. doi:10.1245/s10434-011-1729-9.

19. Ruffini E, Asioli S, Filosso PL, Buffoni L, Bruna MC, Mossetti C, et al. Significance of the presence of microscopic vascular invasion after complete resection of Stage I-II pT1-T2NO non-small cell lung cancer and its relation with T-Size categories: did the 2009 7th edition of the TNM staging system miss something? J Thorac Oncol. 2011;6(2):319-26. doi:10.1097/JTO.0b013e3182011f70.

20. Nitadori J, Bograd AJ, Morales EA, Rizk NP, Dunphy MP, Sima CS, et al. Preoperative consolidation-to-tumor ratio and SUVmax stratify the risk of 
recurrence in patients undergoing limited resection for lung adenocarcinoma $\leq 2 \mathrm{~cm}$. Ann Surg Oncol. 2013;20(13):4282-8. doi:10.1245/s10434-013-3212-2.

21. Tremblay L, Deslauriers J. What is the most practical, optimal, and cost effective method for performing follow-up after lung cancer surgery, and by whom should it be done? Thorac Surg Clin. 2013;23(3):429-36. doi:10.1016/j.thorsurg.2013.05.010

22. Yeh YC, Wu YC, Chen CY, Wang LS, Hsu WH, Chou TY. Stromal invasion and micropapillary pattern in 212 consecutive surgically resected stage I lung adenocarcinomas: histopathological categories for prognosis prediction. J Clin Pathol. 2012;65(10):910-8. doi:10.1136/jclinpath-2012-200882.

23. Woo T, Okudela K, Mitsui H, Tajiri M, Yamamoto T, Rino Y, et al. Prognostic value of the IASLC/ATS/ERS classification of lung adenocarcinoma in stage I disease of Japanese cases. Pathol Int. 2012;62(12):785-91. doi:10.1111/pin.12016.

24. Hung JJ, Jeng WJ, Chou TY, Hsu WH, Wu K, Huang BS, et al. Prognostic value of the new International Association for the Study of Lung Cancer/American Thoracic Society/European Respiratory Society lung adenocarcinoma classification on death and recurrence in completely resected stage I lung adenocarcinoma. Ann Surg. 2013;258(6):1079-86. doi:10.1097/SLA.0b013e31828920c0.

25. Song Z, Zhu H, Guo Z, Wu W, Sun W, Zhang Y. Prognostic value of the IASLC/ ATS/ERS classification in stage I lung adenocarcinoma patients—based on a hospital study in China. Eur J Surg Oncol. 2013;39(11):1262-8. doi:10.1016/ j.ejso.2013.08.026

26. Yanagawa N, Shiono S, Abiko M, Ogata SY, Sato T, Tamura G. New IASLC/ ATS/ERS classification and invasive tumor size are predictive of disease recurrence in stage I lung adenocarcinoma. J Thorac Oncol. 2013;8(5):612-8. doi:10.1097/JTO.0b013e318287c3eb.

27. Ito M, Miyata Y, Kushitani K, Yoshiya T, Mimae T, Ibuki Y, et al. Prediction for prognosis of resected pT1a-1bNOM0 adenocarcinoma based on tumor size and histological status: relationship of TNM and IASLC/ATS/ERS classifications. Lung Cancer. 2014;85(2):270-5. doi:10.1016/j.lungcan.2014.05.014.

\section{Submit your next manuscript to BioMed Central and we will help you at every step:}

- We accept pre-submission inquiries

- Our selector tool helps you to find the most relevant journal

- We provide round the clock customer support

- Convenient online submission

- Thorough peer review

- Inclusion in PubMed and all major indexing services

- Maximum visibility for your research

Submit your manuscript at www.biomedcentral.com/submit

C Biomed Central 